\title{
ON THE EXISTENCE THEOREM FOR DIFFERENTIAL EQUATIONS
}

JOEL W. ROBBIN

In this note we show how to derive the fundamental existence theorem for ordinary differential equations as a corollary of the implicit function theorem in Banach spaces. ${ }^{1}$ The proof of smoothness with respect to initial conditions is considerably shorter than existing proofs (see, for example [3], [4], or [5]). Throughout, a dot (i.e., $\dot{\phi}$ ) denotes differentiation with respect to $t$.

THEOREM. LetUbe an open set in a Banach space E and let $f: R \times U \rightarrow E$ be a $C^{r}$ map $(r \geqq 1)$. Then for each $x_{0} \in U$ there exists an open neighborhood $V$ of $x_{0}$ in $U$, an open interval $(-\epsilon, \epsilon)$ about 0 in $R$ and a map $\phi:(-\epsilon, \epsilon) \times V \rightarrow U$ such that

(1) $\phi$ is $C^{r}$;

(2) $\phi(0, x)=x$ for $x \in V$;

(3) $\dot{\phi}(t, x)=f(t, \phi(t, x))$ for $(t, x) \in(-\epsilon, \epsilon) \times V$.

Proof. We suppose without loss of generality that $x_{0}$ is the origin of $E$ and that $U$ is an open ball with center $x_{0}$. Take $U_{0}$ to be the open ball whose center is $x_{0}$ and whose radius is half the radius of $U$. Let $I$ denote the closed interval $[-1,1] \subseteq R$. For $p$ an integer $\geqq 0$ let $C^{p}(I, E)$ denote the Banach space of $C^{p}$ maps from $I$ to $E$ (with the $C^{p}$ topology), $C_{0}^{p}(I, E)$ be the (closed) subspace of $C^{p}(I, E)$ consisting of all $\gamma \in C^{p}(I, E)$ with $\gamma(0)=0$, and $C_{0}^{p}\left(I, U_{0}\right)$ the set of all $\gamma \in C_{0}^{p}(I, E)$ such that $\gamma(I) \subseteq U_{0}$. Note that $C_{0}^{p}\left(I, U_{0}\right)$ is open in the Banach space $C_{0}^{p}(I, E) . D$ denotes the differentiation operator (see [4] or [5]) and $D_{j}$ denotes partial differentiation with respect to the $j$ th variable.

Let $F: R \times U_{0} \times C_{0}^{1}\left(I, U_{0}\right) \rightarrow C^{0}(I, E)$ be the map defined by

$$
F(a, x, \gamma)(t)=\dot{\gamma}(t)-a f(a t, x+\gamma(t))
$$

for $a \in R, x \in U_{0}, \gamma \in C_{0}^{1}\left(I, U_{0}\right)$ and $t \in I$. One easily verifies that $F$ is a $C^{1}$ map between Banach spaces. (This is an especially easy instance of the so-called omega theorem of [1]. Note that the map $\gamma \rightarrow \dot{\gamma}$ is continuous linear.) The partial derivative with respect to $\gamma$ at the point $a=0, x=x_{0}, \gamma=0$ evaluated at the "tangent vector" $\delta \in C_{0}^{1}(I, E)$ is given by

Received by the editors October 5, 1967.

${ }^{1} \mathrm{I}$ am indebted to R. Abraham for suggesting this to me. 


$$
D_{3} F\left(0, x_{0}, 0\right) \delta(t)=\dot{\delta}(t) ;
$$

it is clearly a toplinear isomorphism. Since $F\left(0, x_{0}, 0\right)=0$ we may apply the implicit function theorem [4, p. 265]. This yields an open neighborhood $(-2 \epsilon, 2 \epsilon) \times V$ of $\left(0, x_{0}\right)$ in $R \times U_{0}$ and a $C^{1} \operatorname{map} H:(-2 \epsilon, 2 \epsilon)$ $\times V \rightarrow C_{0}^{1}\left(I, U_{0}\right)$ such that

$$
F(a, x, H(a, x))=0
$$

for $(a, x) \in(-2 \epsilon, 2 \epsilon) \times V$. We define $\phi:(-\epsilon, \epsilon) \times V \rightarrow U$ by

$$
\phi(t, x)=H(\epsilon, x)(t / \epsilon)+x .
$$

$\phi$ is $C^{1}$ : this follows immediately from the fact that the evaluation map $C_{0}^{1}\left(I, U_{0}\right) \times I \rightarrow U_{0}$ is $C^{1}$ (see $[1]$ or $[2$, p. 25]). $\phi(0, x)=x$ since $H(\epsilon, x) \in C_{0}^{1}\left(I, U_{0}\right)$. Finally, since

$$
\dot{\phi}(t, x)-f(t, \phi(t, x))=(1 / \epsilon) F(\epsilon, x, H(\epsilon, x))(t / \epsilon)=0
$$

it follows that $\phi$ is the solution curve. We have proved the theorem in the case $r=1$. The general case follows from the case $r=1$ by an easy (and standard) induction argument.

\section{BIBLIOGRAPHY}

1. R. Abraham, Lectures of Smale on differential topology, Mimeographed notes, Columbia Univ., New York, 1962.

2. R. Abraham and J. Robbin, Transversality of flows and mappings, Benjamin, New York, 1967.

3. E. A. Coddington and N. Levinson, Theory of ordinary differential equations, McGraw-Hill, New York, 1955. 1960.

4. J. Dieudonné, Foundations of modern analysis, Academic Press, New York,

5. S. Lang, Introduction to differentiable manifolds, Interscience, New York, 1962.

UNIVERSITY OF WISCONSIN 